\title{
The effect of chest compression frequency on the quality of resuscitation by lifeguards. A prospective randomized crossover multicenter simulation trial
}

\author{
Jacek Smereka ${ }^{1}$, Łukasz Iskrzycki ${ }^{1}$, Elżbieta Makomaska-Szaroszyk ${ }^{2}$, Karol Bielski ${ }^{2}$, \\ Michael Frass ${ }^{3}$, Oliver Robak ${ }^{3}$, Kurt Ruetzler ${ }^{4}$, Michael Czekajło, \\ Antonio Rodríguez-Núnez ${ }^{6}$, Jesús López-Herce ${ }^{7}$, Łukasz Szarpak ${ }^{2,5}$
}

${ }^{1}$ Department of Emergency Medical Service, Wroclaw Medical University, Wroclaw, Poland

${ }^{2}$ Lazarski University, Warsaw, Poland

${ }^{3}$ Department of Internal Medicine I, Medical University of Vienna, Austria

${ }^{4}$ Departments of Outcomes Research and General Anesthesia, Cleveland Clinic, Cleveland, OH, USA

${ }^{5}$ Hounter Holmes McGuire Center for Simulation and Healthcare,

Virginia Commonwealth University, Richmond, VA, USA

${ }^{6}$ Pediatric Emergency and Critical Care Division, Pediatric Area Hospital Clinico

Universitario de Santiago de Compostela, Spain

${ }^{7}$ Pediatric Intensive Care Department, Hospital General Universitario Gregorio Marannón,

Medical School, Complutense University of Madrid, Spain

\begin{abstract}
Background: The ability to perform high-quality cardiopulmonary resuscitation is one of the basic skills for lifeguards. The aim of the study was to assess the influence of chest compression frequency on the quality of the parameters of chest compressions performed by lifeguards.

Methods: This prospective observational, randomized, crossover simulation study was performed with 40 lifeguards working in Warsaw, Wroclaw, and Poznan, Poland. The subjects then participated in a target study, in which they were asked to perform 2-min cycles of metronome-guided chest compressions at different rates: 80, 90, 100, 110, 120, 130, 140, and 150 compressions per minute (CPM).

Results: The study involved 40 lifeguards. Optimal chest compression score calculated by manikin software was achieved for 110-120 CPM. Chest compression depth achieved 53 (interquartile range [IQR] 52-54) mm, 56 (IQR 54-57) mm, 52.5 (IQR 50-54) mm, 53 (IQR 52-53) mm, 50 (IQR 49-51) mm, 47 (IQR 44-51) mm, 41 (IQR 40-42) mm, 38 (IQR 38-43) mm for 80, 90, 100, 110, 120, 130, 140 and 150 CPM, respectively. The percentage of chest compressions with the correct depth was lower for rates exceeding 120 CPM.

Conclusions: The rate of 100-120 CPM, as recommended by international guidelines, is the optimal chest compression rate for cardiopulmonary resuscitation performed by lifeguards. A rate above 120 CPM was associated with a dramatic decrease in chest compression depth and overall chest compression quality. The role of full chest recoil should be emphasized in basic life support training. (Cardiol J 2019; 26, 6: 769-776)
\end{abstract}

Key words: chest compression, cardiopulmonary resuscitation, quality, lifeguard, medical simulation

Address for correspondence: Associate Professor, Łukasz Szarpak, PhD, Lazarski University, ul. Świeradowska 43, 02-662 Warszawa, Poland, e-mail: lukasz.szarpak@gmail.com 


\section{Introduction}

An ability to perform cardiopulmonary resuscitation is one of the basic skills that lifeguards should possess [1,2]. According to numerous studies, high-quality chest compression has a direct impact on the effectiveness for the return of spontaneous circulation [3, 4]. Current cardiopulmonary resuscitation guidelines of both the European Resuscitation Council (ERC) and the American Heart Association (AHA) emphasize the need to minimize interruptions in chest compressions and to perform high-quality chest compressions as determined by the correct rate and depth of compressions, full chest recoil, and appropriate positioning of hands on the chest [5-7]. All these factors have a significant impact on the effectiveness of cardiopulmonary resuscitation. The ERC and AHA guidelines recommend that chest compressions should be performed at a rate of 100-120 compressions per minute (CPM). However, there is no clear consensus that would indicate an optimal chest compression frequency for adults and children [8].

The aim of the study was to assess the influence of chest compression frequency on the quality of parameters of chest compressions performed by lifeguards.

\section{Methods}

\section{Setting and participants}

The study was designed as a prospective observational, randomized, crossover simulation study. The study protocol was approved by the institutional review board of the Polish Society of Disaster Medicine (approval No., 19.02.2018.IRB). The survey was conducted among lifeguards working in Warsaw, Wroclaw, and Poznan, Poland. Being an active lifeguard and a voluntary participant in the survey constituted criteria for inclusion. Among exclusion criteria were: failure to meet the inclusion criteria, as well as back pain or upper limb pain or injury that would prevent performing chest compressions. Written voluntary informed consent was obtained from each participant.

\section{Material and methods}

In order to simulate a patient requiring cardiopulmonary resuscitation, an adult simulator, the Resusci Anne Simulator (Laerdal, Stavanger, Norway) was utilized and placed on a flat surface in a brightly lit room $[9,10]$. The simulator was equipped with a SimPad PLUS device (Laerdal, Stavanger, Norway), which allowed for control of the simulator and record parameters related to the resuscitation procedure.

Before entering the study, all subjects participated in a course on basic life support (BLS) conducted by AHA instructors. The training was based on the AHA 2015 guidelines [5].

The lifeguards then participated in the targeted study in which they were asked to perform 2 -min cycles of chest compressions at different rates: $80,90,100,110,120,130,140$, and $150 \mathrm{CPM}$.

For this purpose, a metronome was used, which was set at the appropriate frequency. After each 2 -min cycle of compressions at a certain rate, the participants had a 20-min break after which they performed compressions at a different rate. The order of both the participants and the frequency of chest compressions was random. The Research Randomizer software (www.randomizer. org) was used for this purpose.

\section{Data collection and analysis}

The parameters recorded by the SimPad PLUS software were analyzed. The frequency of chest compressions, chest compression fraction, mean time of breaks in chest compressions, correct hand position, chest compression depth, full chest recoil, and proportion of compressions with appropriate depth were studied. Chest compression fraction was defined as the percentage of time during which chest compressions were performed. Chest compression score $(0-100 \%)$ was calculated by manikin software on the basis of the following parameters: compression depth, compression rate, share of incomplete chest recoil, number of compressions per cycle, and correct hand position on the manikin's chest. According to resuscitation guidelines, the depth of chest compressions should equal 50-60 mm.

Additionally, background information was recorded about the participants, including their age, sex, and work experience.

\section{Statistical analysis}

All statistical analysis was performed with Statistica 13.1EN for Windows statistical package (TIBCO Software Inc, Tulsa, OK, USA). Data were described as percentages or medians and interquartile ranges (IQR). Non-parametric tests were applied when data distribution was not normal, as implied by the Shapiro-Wilk and KolmogorovSmirnov tests. Values of $\mathrm{p}<0.05$ were considered significant. 


\section{Results}

The study included 40 lifeguards (14 females, $35 \%$ ), whose median age was 25.6 (IQR 23-32) years, and median work experience equaled 6.5 (IQR 2-10) years.

The chest compression parameters, including chest compression score, chest compression fraction, no flow time, correct hand position, chest compression depth, full chest recoil, and the proportion of compressions with correct depth for different chest compression rates are shown in Table 1.

The overall chest compression score (Fig. 1) achieved 62 (IQR 58-65), 84 (IQR 82-88), 92 (IQR 87-93), 93.5 (IQR 91.5-97), 73 (IQR 71.5-80), 67 (IQR 64-69), 34 (IQR 29-39.5), 19 (IQR 18-21) for $80,90,100,110,120,130,140$ and $150 \mathrm{CPM}$, respectively.

Median chest compression fraction (Fig. 2) was $99 \%$ for $80 \mathrm{CPM}$ and $100 \%$ all $90-150 \mathrm{CPM}$ and no flow time was $0 \mathrm{~s}$ was for all studied chest compression rates. The percentage of chest compressions with correct hand position achieved 100 (IQR 100-100), 100 (IQR 99-100), 100 (IQR 99-100), 99 (IQR 98-100), 100 (IQR 99-100), 99 (IQR 98-100), 97 (IQR 96-99.5), 98 (IQR 96-100) for $80,90,100,110,120,130,140$ and $150 \mathrm{CPM}$, respectively.

Chest compression depth (Fig. 3) was 53 (IQR 52-54) mm, 56 (IQR 54-57) mm, 52.5 (IQR 50-54) $\mathrm{mm}, 53$ (IQR 52-53) mm, 50 (IQR 49-51) mm, 47 (IQR 44-51) mm, 41 (IQR 40-42) mm, 38 (IQR 38-43) $\mathrm{mm}$ for $80,90,100,110,120,130,140$ and 150 CPM, respectively.

The percentage of compression with full chest recoil (Fig. 4) achieved 71 (IQR 49-76), 28 (IQR 14-41.5), 39 (IQR 18-44), 34.5 (31-40.5), 11 (IQR 9-21), 16 (IQR 7-15), 17.5 (IQR 16-20), 13 (IQR 11-16) for $80,90,100,110,120,130,140$ and 150 CPM, respectively.

\section{Discussion}

Lifeguards fulfil their duties at swimming pools and on beaches, and can also perform cardiopulmonary resuscitation as bystanders in emergency situations [11-13]. They are obliged to participate in courses on BLS and automated external defibrillators (AED) on a regular basis [14]. There are several factors influencing high-quality adult chest compression, including trunk and arm muscle mass, basal metabolic rate, mean fat-free mass, and other individual parameters [15-18]. Lifeguards

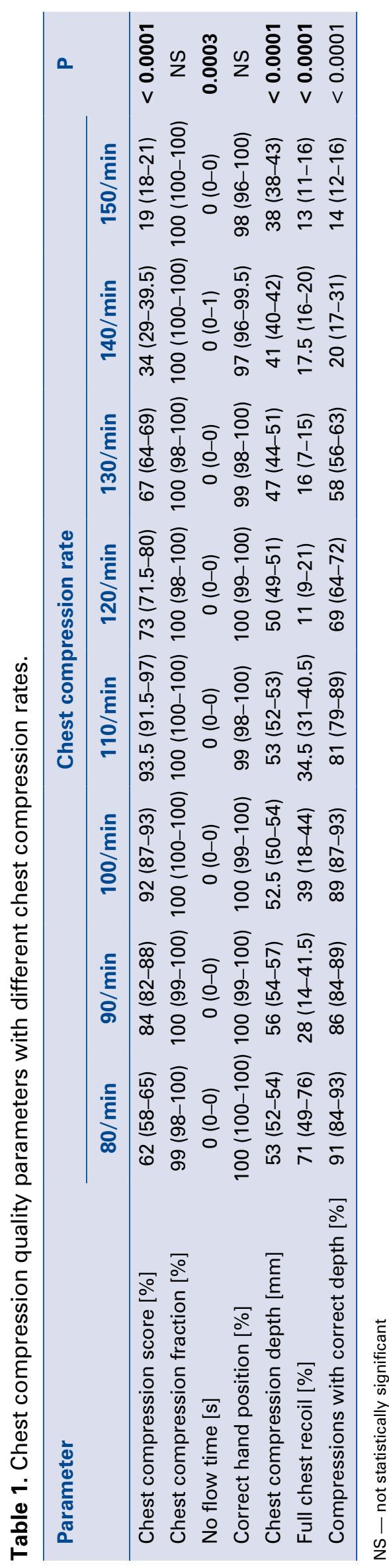


Cardiology Journal 2019, Vol. 26, No. 6

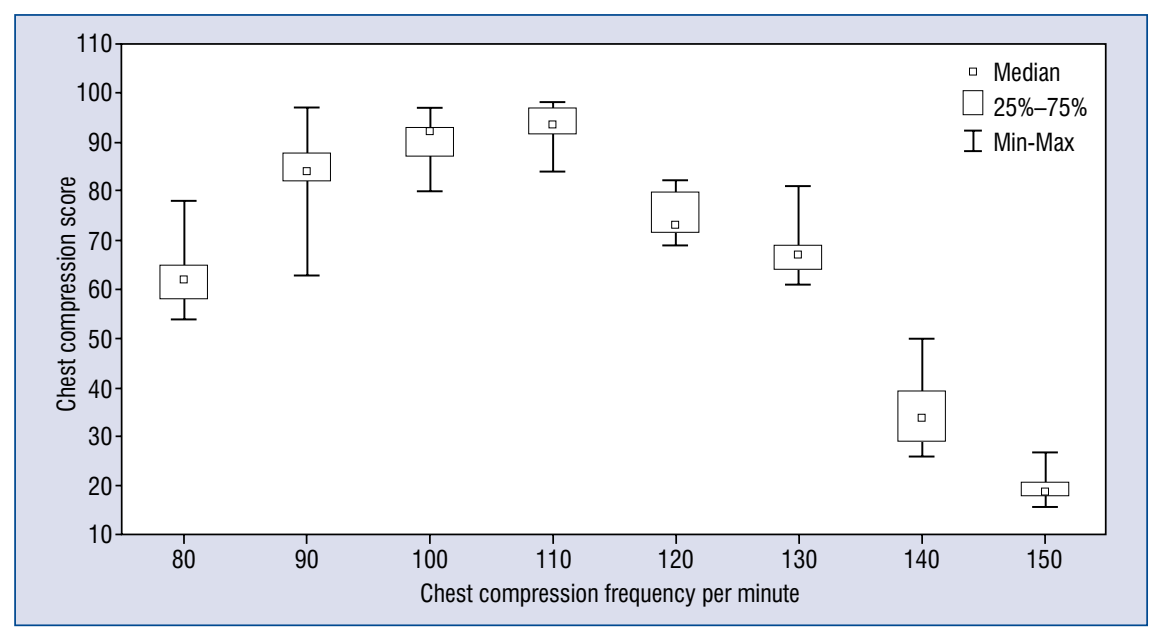

Figure 1. Chest compressions score.

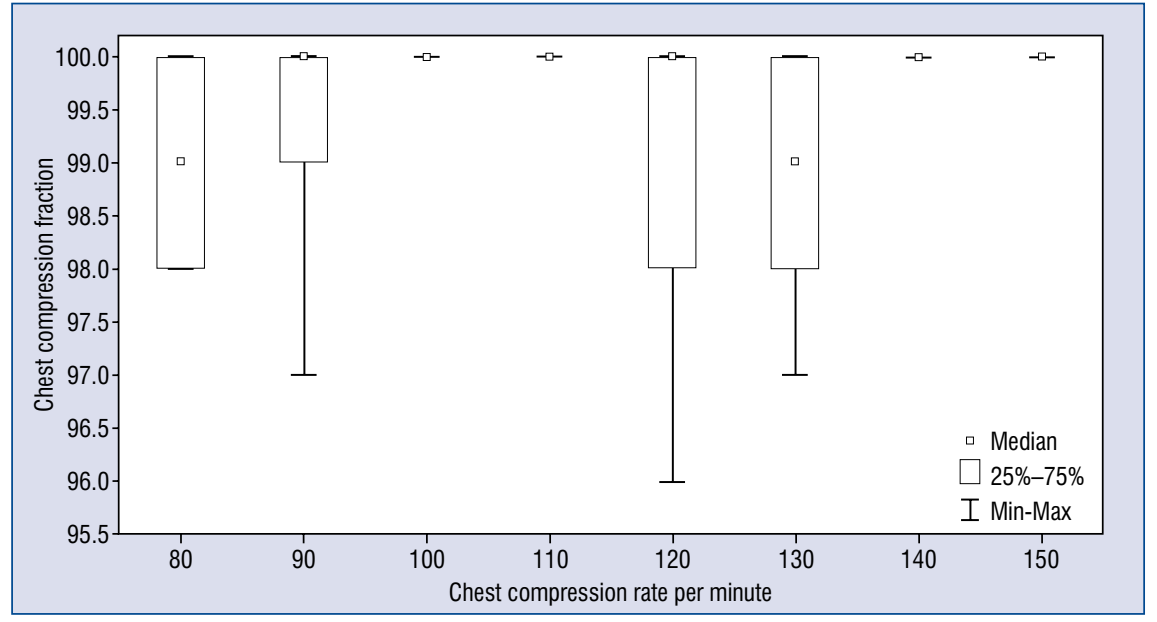

Figure 2. Chest compression fraction.

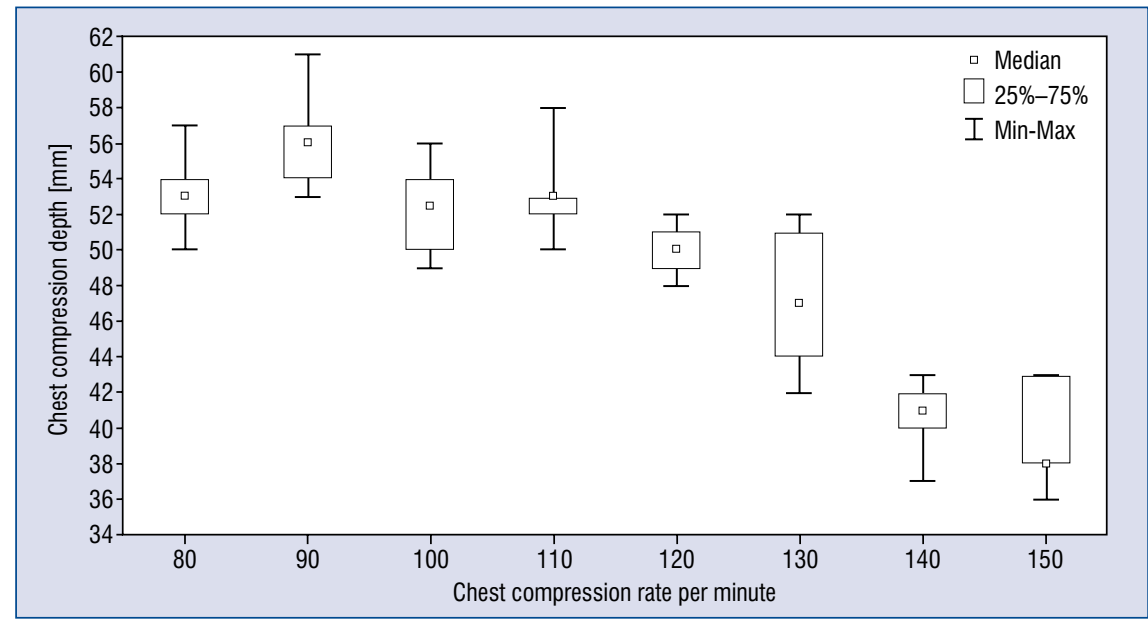

Figure 3. Chest compression depth. 


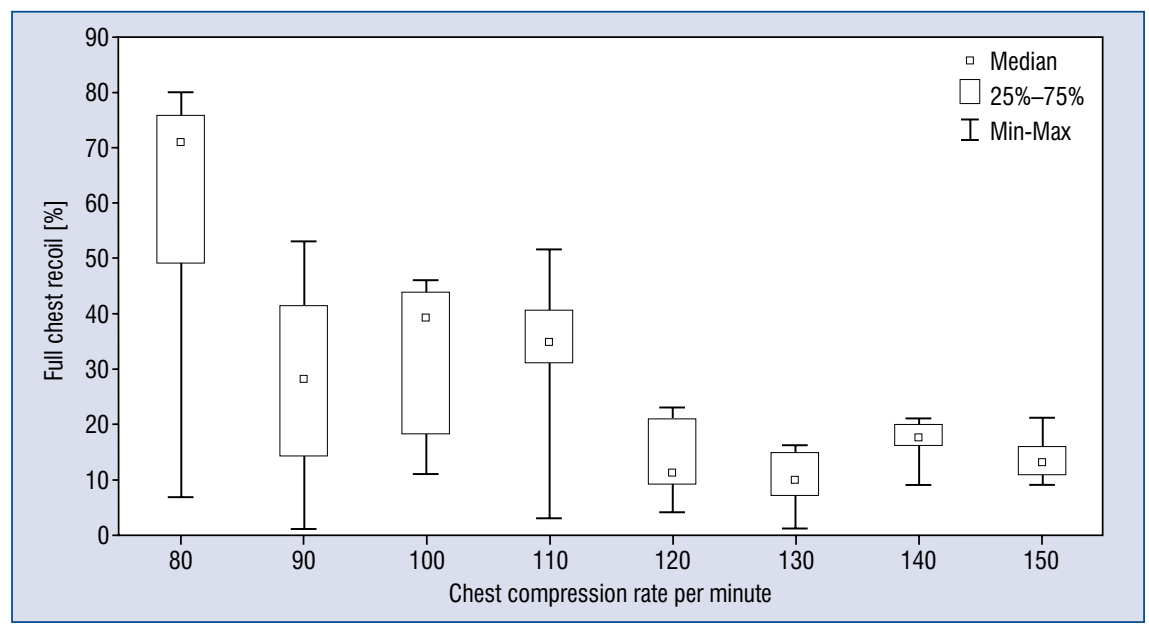

Figure 4. Percentage of full chest recoil.

often meet optimal criteria for body mass index and mean fat-free mass, therefore it can be expected that resuscitation performed by this professional group should be of a high-quality; however, water rescue actions are exhausting and can impede the quality of resuscitation. The quality of chest compression worsens after a water rescue action by 26-28\%, so it has been emphasized that the use of additional equipment (fins and rescue tubes) provides benefit in emergency situations [19]. Some publications suggest that lifesavers clear out blood lactate more efficiently when performing an active recovery protocol [20]. The quality of cardiopulmonary resuscitation performed by lifeguards using a CPRmeter monitor improved significantly in the feedback group, compared with the non-feedback group [2]. Chest compressions on inflatable rescue boats (IRBs) were analyzed by Barcala-Furelos et al. [21] and it was suggested that surf-lifeguards could deliver good-quality resuscitation even on a moving IRB, although their performance is worse than onshore.

Cardiopulmonary resuscitation in a lifeguard's daily practice does not occur often, but the quality of chest compressions performed in victims can impede their neurological outcome. In the analysis of interventions undertaken by lifeguards in Brazil, Szpilman et al. [22] revealed that resuscitation was performed rarely and took place for only 1 in every 112,000 lifeguarding actions $(0.0009 \%)$.

In the present study, it was observed that chest compression rate impeded chest compression quality parameters, including chest compression depth and complete chest recoil, as well as the number of compressions per cycle. The optimal chest compression score was achieved for the rate of
100-110 CPM. Chest compression depth was within the resuscitation guidelines for the rate of chest compressions not exceeding $120 \mathrm{CPM}$. The percentage of full chest recoil was low for all chest compression rates, excluding the lowest one at $80 \mathrm{CPM}$, and especially for rates above $110 \mathrm{CPM}$ which were associated with very low full chest recoil percentage $(<18 \%)$. Accurate chest compression depth assessment is difficult for healthcare professionals during cardiopulmonary resuscitation. When performing chest compressions, it is possible to precisely differentiate $0.5-\mathrm{cm}$ differences in the compression depth but not possible to accurately determine overall target depth [23].

In a prospective observational study at a single academic medical center carried out by Kilgannon et al. [24], it was suggested that chest compression rates of 121-140 CPM were bound with the highest odds ratio of return of spontaneous circulation (ROSC), and the rates exceeding the currently recommended 100-120 CPM might improve the chances for ROSC among in-hospital cardiac arrest patients. This study was based on resuscitation preformed in a near-ideal in-hospital setting in intubated patients, and several factors could have influenced the results.

Lee et al. [25] analyzed chest compression parameters in 322 students participating in a cardiopulmonary resuscitation contest. The authors noticed that chest compression depth was proportional to chest compression rate, though with significantly more incomplete chest recoils at a rate of more than $120 \mathrm{CPM}$.

Zou et al. [26] dealt with 2-min chest compression-only resuscitation with guiding sounds at three rates $(100,120$, and $140 \mathrm{CPM})$ in a random 
sequence. They noticed that the complete chest compression release and fractions of chest compressions with sufficient depth were deteriorated at a rate of $140 \mathrm{CPM}$.

The impact of chest compression rate on survival outcome has been analyzed in several publications. Sutton et al. [27] studied the influence of compliance with guidelines referring to chest compression rate during pediatric in-hospital resuscitation on survival outcomes. The chest compression rate within the recommended range was associated with a slightly better outcome. Fernando et al. [28] analyzed out-of-hospital cardiac arrest cases and the quality of cardiopulmonary resuscitation performed by bystanders using AED on the basis of data stored by the defibrillator. They concluded that bystanders performed high-quality resuscitation, compliant with the international guidelines, especially during the first $5 \mathrm{~min}$ (but not in the first minute).

Chest compression depth is affected by chest compression rate even during metronome-guided simulated manikin resuscitation [29]. In a simulation study concerning adult cardiopulmonary resuscitation performed with the use of a metronome at different frequencies $(80,100,120$, and 140 ticks/ $/ \mathrm{min}$ ), the average compression depth increased when the metronome rate increased, and with the metronome rates of 80 and 100 ticks/min it was significantly lower than in procedures without metronome guidance [30].

The achievement of the target chest compression depth and rate, within the international guidelines recommendation, is difficult for many laypersons; however, the use of feedback/prompt devices significantly improves the quality of handsonly resuscitation [31, 32].

Maier et al. [33], in an article published in 1986 in "Circulation", based on animal studies, suggested that the optimal chest compression rate for systemic and coronary perfusion was $120 \mathrm{CPM}$.

In a study published in 2016, Lee et al. [34] analyzed metronome-guided adult continuous chest compressions with the metronome set to 100,120 , 140 , and $160 \mathrm{bpm}$, in a random order. They revealed that the share of incomplete chest recoils was lower at the rates of 100 and $120 \mathrm{CPM}$ as compared with 160 CPM. Most importantly, the share of chest compressions fulfilling the criteria for high-quality resuscitation at the rate of $120 \mathrm{CPM}$ was significantly higher than at $100 \mathrm{CPM}$.

Idris et al. [35] suggested in an article published in 2012 in "Circulation" that the chest compression rate was associated with ROSC but not with survival to hospital discharge in out-of-hospital cardiac arrest patients. In 2015, Idris et al. [36] analyzed data abstracted from monitor-defibrillator recordings for the first 5 min of emergency medical service in adult cardiopulmonary resuscitation. They concluded that after adjustment for chest compression fraction and depth, compression rates of 100-120 CPM were associated with greatest survival to hospital discharge.

A meta-analysis by Taliowska et al. [37] concerning the impact of cardiopulmonary resuscitation quality parameters, including chest compression depth and rate, on patient survival from cardiac arrest revealed that chest compression depth and rate were associated with survival outcomes.

Field et al. [38] published a study comparing chest compression rates of $80,100,120,140$, and $160 \mathrm{CPM}$ during simulated cardiopulmonary resuscitation in adults. Higher chest compression rates were associated with reduced chest compression depth $(39.5 \mathrm{~mm}$ at $80 \mathrm{CPM}$ vs. $34.5 \mathrm{~mm}$ at $160 \mathrm{CPM}$, $\mathrm{p}<0.001)$. The final conclusion was that rates above 120 CPM were bound with the greatest reduction of chest compression quality.

Monsieurs et al. [39] analyzed chest compression rate and depth recorded with an accelerometer in out-of-hospital cardiac arrest patients. Of all compressions, $36 \%$ were performed at a rate exceeding $120 \mathrm{CPM}$, and only $19 \%$ reached the depth of more than $5 \mathrm{~cm}$. In $58 \%$ of patients, a statistically significantly lower depth was observed for chest compression rates above $120 \mathrm{CPM}$ compared with 80-120 CPM. The study has concluded that higher compression rates were associated with lower compression depths.

\section{Limitations of the study}

There are several limitations in the present study. The study was performed using manikin models with all the limitations for this type of research, however in resuscitation trials, the use of simulators is standard procedure and enables equal and repetitive conditions for all participants [40-43]. The second limitation is the study group. This study analyzed only lifeguards and results may not be similar in other professions, medical personnel or laypersons. However, lifeguards have generally very good health status and are in first line during out-of-hospital cardiac arrest resuscitations. The next limitation is chest compression time. In the present study, a 2 -min resuscitation cycle was evaluated, but different values could be obtained during longer resuscitations, such as over a 10-min period. However, it should be noted that 
the ERC recommends changing rescuer after about 2 min of chest compression, hence a 2 -min cycle herein seems to be justified.

\section{Conclusions}

The chest compression rate of 100-120 CPM, recommended by international guidelines, is optimal for cardiopulmonary resuscitation performed by lifeguards. Rates above $120 \mathrm{CPM}$ are associated with a dramatic decrease in chest compression depth and overall chest compression quality. The role of full chest recoil should be emphasized in BLS training.

\section{Conflict of interest: None declared}

\section{References}

1. Pakula RJ, Wanat S. CPR in terms of maritime search and rescue service working conditions. Disaster Emerg Med J. 2017; 2(2): 104-105, doi: 10.5603/DEMJ.2017.0022.

2. Iskrzycki L, Smereka J, Rodriguez-Nunez A, et al. The impact of the use of a CPRMeter monitor on quality of chest compressions: a prospective randomised trial, cross-simulation. Kardiol Pol. 2018; 76(3): 574-579, doi: 10.5603/KP.a2017.0255, indexed in Pubmed: 29297195.

3. Aleksandrowicz S, Madziala M, Iskrzycki L, et al. Performance of chest compressions with the use of the new mechanical chest compression machine lifeline arm: a randomized crossover manikin study in novice physicians. Disaster Emerg Med J. 2016; 1(1): 30-36, doi: 10.5603/DEMJ.2016.0005.

4. Smereka J, Kasiński M, Smereka A, et al. The quality of a newly developed infant chest compression method applied by paramedics: a randomised crossover manikin trial. Kardiol Pol. 2017; 75(6): 589-595, doi: 10.5603/KP.a2017.0015, indexed in Pubmed: 28150278 .

5. Kleinman ME, Brennan EE, Goldberger ZD, et al. Part 5: Adult Basic Life Support and Cardiopulmonary Resuscitation Quality: 2015 American Heart Association Guidelines Update for Cardiopulmonary Resuscitation and Emergency Cardiovascular Care. Circulation. 2015; 132(18 Suppl 2): S414-S435, doi: 10.1161/ /CIR.0000000000000259, indexed in Pubmed: 26472993.

6. Truszewski Z, Szarpak L, Kurowski A, et al. Randomized trial of the chest compressions effectiveness comparing 3 feedback CPR devices and standard basic life support by nurses. Am J Emerg Med. 2016; 34(3): 381-385, doi: 10.1016/j.ajem.2015.11.003, indexed in Pubmed: 26612703.

7. Smereka J, Szarpak L, Rodríguez-Núñez A, et al. A randomized comparison of three chest compression techniques and associated hemodynamic effect during infant CPR: A randomized manikin study. Am J Emerg Med. 2017; 35(10): 1420-1425, doi: 10.1016/j.ajem.2017.04.024, indexed in Pubmed: 28433454.

8. Wieczorek W, Kaminska H. Impact of a corpuls CPR mechanical chest compression device on chest compression quality during extended pediatric manikin resuscitation: a randomized crossover pilot study. Disaster Emerg Med J. 2017; 2(2): 58-63, doi: 10.5603/DEMJ.2017.0012.
9. Czekajlo M, Dabrowska A. In situ simulation of cardiac arrest. Disaster Emerg Med J. 2017; 2(3): 116-119, doi: 10.5603/ /DEMJ.2017.0025.

10. Abelsson A. Learning through simulation. Disaster Emerg Med J. 2017; 2(3): 125-128, doi: 10.5603/DEMJ.2017.0027.

11. Freund B, Kaplan PW. A review of the utility of a hypothermia protocol in cardiac arrests due to non-shockable rhythms. Cardiol J. 2017; 24(3): 324-333, doi: 10.5603/CJ.a2017.0016, indexed in Pubmed: 28150290

12. Claesson A, Karlsson T, Thorén AB, et al. Delay and performance of cardiopulmonary resuscitation in surf lifeguards after simulated cardiac arrest due to drowning. Am J Emerg Med. 2011; 29(9): 1044-1050, doi: 10.1016/j.ajem.2010.06.026, indexed in Pubmed: 20870373.

13. Kurowski A, Szarpak $€$, Bogdański $€$, et al. Comparison of the effectiveness of cardiopulmonary resuscitation with standard manual chest compressions and the use of TrueCPR and PocketCPR feedback devices. Kardiol Pol. 2015; 73(10): 924-930, doi: 10.5603/KP.a2015.0084, indexed in Pubmed: 25985725.

14. Telec W, Baszko A, Dąbrowski M, et al. Automated external defibrillator use in public places: a study of acquisition time. Kardiol Pol. 2018; 76(1): 181-185, doi: 10.5603/KP.a2017.0199, indexed in Pubmed: 29168550.

15. Abelairas-Gómez C, Barcala-Furelos R, Szarpak $\mathrm{E}$, et al. The effect of strength training on quality of prolonged basic cardiopulmonary resuscitation. Kardiol Pol. 2017; 75(1): 21-27, doi: 10.5603/KP.a2016.0165, indexed in Pubmed: 27878801.

16. Abelairas-Gómez C, Barcala-Furelos R, Szarpak $\mathrm{E}$, et al. Response to the letter concerning the article:. Kardiol Pol. 2017; 75(1): 88-89, doi:10.5603/KP.2017.0011, indexed in Pubmed: 28124792 .

17. Kaminska H, Wieczorek W, Matusik P, et al. Factors influencing high-quality chest compressions during cardiopulmonary resuscitation scenario, according to 2015 American Heart Association Guidelines. Kardiol Pol. 2018; 76(3): 642-647, doi: 10.5603/ /KP.a2018.0003, indexed in Pubmed: 29313566.

18. Jorge-Soto C, Abilleira-González M, Otero-Agra M, et al. Schoolteachers as candidates to be basic life support trainers: A simulation trial. Cardiol J. 2019; 26(5): 536-542, doi: 10.5603/ /CJ.a2018.0073, indexed in Pubmed: 30009374.

19. Abelairas-Gómez C, Barcala-Furelos R, Mecías-Calvo M, et al. Prehospital Emergency Medicine at the Beach: What Is the Effect of Fins and Rescue Tubes in Lifesaving and Cardiopulmonary Resuscitation After Rescue? Wilderness Environ Med. 2017; 28(3): 176-184, doi: 10.1016/j.wem.2017.03.013, indexed in Pubmed: 28754294.

20. Kalén A, Pérez-Ferreirós A, Barcala-Furelos R, et al. How can lifeguards recover better? A cross-over study comparing resting, running, and foam rolling. Am J Emerg Med. 2017; 35(12): 1887-1891, doi: 10.1016/j.ajem.2017.06.028, indexed in Pubmed: 28651888.

21. Barcala-Furelos R, Abelairas-Gomez C, Palacios-Aguilar J, et al. Can surf-lifeguards perform a quality cardiopulmonary resuscitation sailing on a lifeboat? A quasi-experimental study. Emerg MedJ. 2017; 34(6): 370-375, doi: 10.1136/emermed-2016-205952, indexed in Pubmed: 28130348.

22. Szpilman D, de Barros Oliveira R, Mocellin O, et al. Is drowning a mere matter of resuscitation? Resuscitation. 2018; 129: 103-106, doi: 10.1016/j.resuscitation.2018.06.018, indexed in Pubmed: 29928958. 
23. Deakin CD, Sidebottom DB, Potter R. Can rescuers accurately deliver subtle changes to chest compression depth if recommended by future guidelines? Resuscitation. 2018; 124: 58-62, doi: 10.1016/j.resuscitation.2018.01.010, indexed in Pubmed: 29309883.

24. Kilgannon JH, Kirchhoff M, Pierce L, et al. Association between chest compression rates and clinical outcomes following in-hospital cardiac arrest at an academic tertiary hospital. Resuscitation. 2017; 110: 154-161, doi: 10.1016/j.resuscitation.2016.09.015, indexed in Pubmed: 27666168.

25. Lee SH, Kim K, Lee JH, et al. Does the quality of chest compressions deteriorate when the chest compression rate is above 120/min? Emerg Med J. 2014; 31(8): 645-648, doi: 10.1136/ /emermed-2013-202682, indexed in Pubmed: 23704754.

26. Zou Y, Shi W, Zhu Y, et al. Rate at 120 /min provides qualified chest compression during cardiopulmonary resuscitation. Am J Emerg Med. 2015; 33(4): 535-538, doi: 10.1016/j.ajem.2015.01.024, indexed in Pubmed: 25662803.

27. Sutton RM, Reeder RW, Landis W, et al. Chest compression rates and pediatric in-hospital cardiac arrest survival outcomes. Resuscitation. 2018; 130: 159-166, doi: 10.1016/j.resuscitation.2018.07.015, indexed in Pubmed: 30031055.

28. Fernando SM, Vaillancourt C, Morrow S, et al. Analysis of bystander CPR quality during out-of-hospital cardiac arrest using data derived from automated external defibrillators. Resuscitation. 2018; 128: 138-143, doi: 10.1016/j.resuscitation.2018.05.012, indexed in Pubmed: 29753856.

29. Bae J, Chung TN, Je SMo. Effect of the rate of chest compression familiarised in previous training on the depth of chest compression during metronome-guided cardiopulmonary resuscitation: a randomised crossover trial. BMJ Open. 2016; 6(2): e010873, doi: 10.1136/bmjopen-2015-010873, indexed in Pubmed: 26873050.

30. Chung TN, Kim SW, You JeS, et al. A higher chest compression rate may be necessary for metronome-guided cardiopulmonary resuscitation. Am J Emerg Med. 2012; 30(1): 226-230, doi: 10.1016/j.ajem.2010.11.026, indexed in Pubmed: 21208766.

31. Liu Y, Huang Z, Li H, et al. CPR feedback/prompt device improves the quality of hands-only $\mathrm{CPR}$ performed in manikin by laypersons following the 2015 AHA guidelines. Am J Emerg Med. 2018 [Epub ahead of print], doi: 10.1016/j.ajem.2018.02.034, indexed in Pubmed: 29525478.

32. Treptau J, Ebnet J, Akin M, et al. Angiographic detection of fatal acute aortic dissection Stanford type A under resuscitation. Cardiol J. 2016; 23(6): 620-622, doi: 10.5603/CJ.2016.0103, indexed in Pubmed: 27976792.

33. Maier GW, Newton JR, et al. Jr, Wolfe JA, The influence of manual chest compression rate on hemodynamic support during cardiac arrest: high-impulse cardiopulmonary resuscitation. Circulation. 1986; 74(6 Pt 2): IV51-IV59.
34. Lee SH, Ryu JiHo, Min MKi, et al. Optimal chest compression rate in cardiopulmonary resuscitation: a prospective, randomized crossover study using a manikin model. Eur J Emerg Med. 2016; 23(4): 253-257, doi: 10.1097/MEJ.0000000000000249, indexed in Pubmed: 25710082.

35. Idris AH, Guffey D, Pepe PE, et al. Relationship between chest compression rates and outcomes from cardiac arrest. Circulation. 2012; 125(24): 3004-3012, doi: 10.1161/CIRCULATIONAHA.111.059535, indexed in Pubmed: 22623717.

36. Idris AH, Guffey D, Pepe PE, et al. Chest compression rates and survival following out-of-hospital cardiac arrest. Crit Care Med. 2015; 43(4): 840-848, doi: 10.1097/CCM.0000000000000824, indexed in Pubmed: 25565457.

37. Talikowska M, Tohira H, Finn J. Cardiopulmonary resuscitation quality and patient survival outcome in cardiac arrest: A systematic review and meta-analysis. Resuscitation. 2015; 96: 66-77, doi: 10.1016/j.resuscitation.2015.07.036, indexed in Pubmed: 26247143.

38. Field RA, Soar J, Davies RP, et al. The impact of chest compression rates on quality of chest compressions - a manikin study. Resuscitation. 2012; 83(3): 360-364, doi: 10.1016/j.resuscitation.2011.07.012, indexed in Pubmed: 21771570.

39. Monsieurs KG, De Regge M, Vansteelandt K, et al. Excessive chest compression rate is associated with insufficient compression depth in prehospital cardiac arrest. Resuscitation. 2012; 83(11): 1319-1323, doi: 10.1016/j.resuscitation.2012.07.015, indexed in Pubmed: 22828356.

40. Truszewski Z, Szarpak $€$, Smereka J, et al. Comparison of the VivaSight single lumen endotracheal tube and the Macintosh laryngoscope for emergency intubation by experienced paramedics in a standardized airway manikin with restricted access: a randomized, crossover trial. Am J Emerg Med. 2016; 34(5): 929-930, doi: 10.1016/j.ajem.2016.02.054, indexed in Pubmed: 26979260.

41. Smereka J, Bielski K, Ladny JR, et al. Evaluation of a newly developed infant chest compression technique: A randomized crossover manikin trial. Medicine (Baltimore). 2017; 96(14): e5915, doi: 10.1097/MD.0000000000005915, indexed in Pubmed: 28383397.

42. Ladny JR, Smereka J, Szarpak L. Comparison of the Trachway video intubating stylet and Macintosh laryngoscope for endotracheal intubation. Preliminary data. Am J Emerg Med. 2017; 35(4): 574-575, doi: 10.1016/j.ajem.2016.12.015, indexed in Pubmed: 27986336.

43. Szarpak $€$, Truszewski Z, Smereka J, et al. Does the use of a chest compression system in children improve the effectiveness of chest compressions? A randomised crossover simulation pilot study. Kardiol Pol. 2016; 74(12): 1499-1504, doi: 10.5603/ /KP.a2016.0107, indexed in Pubmed: 27391911. 